\title{
Perfil do bebê e familiares assistidos em um Ambulatório de Seguimento de Prematuros
}

\section{Profile of assisted baby and family in a Premature Follow-up Ambulatory}

\author{
Larissa Ten Caten Froehlich, Nathalie da Costa Nascimento, Dani Laura Peruzzolo, \\ Vitória Hoerbe Beltrame, Anaelena Bragança de Moraes
}

Como citar este artigo: FROEHLICH, LARISSA T. C.: NASCIMENTO, NATHALIE C.: PERUZZOLO, DANI L.; BELTRAME, VITÓRIA H.; MORAES, ANAELENA B. Perfil do bebê e familiares assistidos em um Ambulatório de Seguimento de Prematuros. Revista Saúde (Sta. Maria). 2020; 46 (2).

\section{Autor correspondente:} Nome: Larissa Ten Caten Froehlich E-mail: larissa_tcf@hotmail.com Telefone: (55) 98123-0093 Formação Profissional: Graduada em Terapia Ocupacional pela Universidade Federal de Santa Maria (UFSM), Santa Maria, RS, País, Brasil.

Filiação Institucional: Universidade Federal de Santa Maria

Endereço para correspondência: Avenida Roraima n : 1000

Bairro: Camobi

Cidade: Santa Maria

Estado: Rio Grande do Sul

CEP: 97 105-600

Data de Submissão:

09/01/2020

Data de aceite:

02/06/2020

Conflito de Interesse: Não há conflito de interesse

\section{(cc) $\mathrm{BY}-\mathrm{NC}-\mathrm{ND}$}

\section{RESUMO}

Objetivo: Traçar o perfil do bebê prematuro e família atendidos em um Programa de Seguimento de Prematuros de um hospital universitário no interior do Rio Grande do Sul. Método: Estudo quantitativo, longitudinal, de análise descritiva realizada com 92 bebês entre os anos de 2013 a 2015. Resultados: $54,3 \%$ nasceram de parto cesáreo, 58,7\% eram meninas. 58,7\% prematuros moderados a tardio. $43,5 \%$ possuíam baixo peso ao nascer e $40,2 \%$ muito baixo peso. Dos bebês recém nascidos de baixo peso $77,5 \%$ foram classificados como prematuros moderados a tardios. Das famílias, $57,1 \%$ residem na cidade do hospital; $56,5 \%$ vivem em casa própria; $68,2 \%$ estavam em união estável. Quanto aos cuidados do bebê, $42,0 \%$ eram feitos pelo pai e mãe. As mães tiveram idade significativamente menor frente a idade dos pais. Houve maior número de pais com emprego formal, e as mães apresentaram maior grau de escolaridade. Conclusão: Existem diferentes perfis de bebês e familiares que devem ser considerados no planejamento/execução de políticas públicas que acolham as necessidades locais através da formação continuada de profissionais e no acolhimento, educação e informação dos pais quanto a seus direitos e responsabilidade, assegurados pelo Sistema Único de Saúde.

PALAVRAS-CHAVE: Recém-nascido prematuro; Crescimento e desenvolvimento; Serviços de Saúde da Criança.

\section{ABSTRACT}

Objective: To trace the profile of the premature baby and family attended in the Premature Followup Program of a university hospital in the interior of Rio Grande do Sul. Methodology: Quantitative, longitudinal study, of descriptive analysis performed with 92 babies between the years 2013 to 2015 . Results and Discussion: $54.3 \%$ were born cesarean birth. $58.7 \%$ were girls. $58.7 \%$ are moderate premature to late premature. $43.5 \%$ had low birth weight and $40.2 \%$ had very low birth weight. $77.5 \%$ of newborn babies of low birth weight were classified as moderate to late premature. About the families, $57.1 \%$ reside in the city of the hospital; $56.5 \%$ live in their own homes; $68.2 \%$ were in common-law marriage. As for the care of the baby, $42.0 \%$ were made by the father and mother. Mothers were significantly younger than the fathers. There were more fathers with formal employment, and mothers had higher education levels. Conclusion: There are different profiles of babies and family members that should be considered in the planning/implementation of public policies that meet the local needs through the continuous training of professionals and in the reception, education and information of the parents regarding their rights and responsibility, guaranteed by the Single System of Health.

KEYWORDS: Premature; Growth and development; Child Health Services. 


\section{INTRODUÇÃO}

A prematuridade neonatal é considerada uma das maiores causas de morte neonatal entre crianças menores de cinco anos. A Organização Mundial da Saúde (OMS) define a morte neonatal como aquela que ocorre durante o primeiro mês de vida e prematura a criança nascida de uma gestação com tempo inferior a 37 semanas ${ }^{1}$. Os bebês prematuros estão propensos a apresentar alterações no desenvolvimento motor, cognitivo, sensorial, funcional e de linguagem, necessitando de acompanhamento diferenciado, pois mesmo após a alta hospitalar, continuam sendo considerados de risco ${ }^{2,3,4}$.

O estado do Rio Grande do Sul apresentou em 2014, 11,4\% de partos prematuros, segundo o Plano Estadual de Saúde ${ }^{5}$, sendo esse percentual maior em relação aos anos anteriores e acima dos $10 \%$ que a OMS recomenda. Em vista disso, é importante garantir o investimento em políticas públicas que desenvolvam ações qualificadas para a assistência imediata e longitudinal às mães e seus bebês prematuros. No Brasil, os Programas de Seguimento de Prematuros (PSP) egressos das Unidades de Tratamento Intensivo Neonatal (UTIN), também chamados de follow-up, foram instituídos com o objetivo de diminuir o impacto da prematuridade no desenvolvimento infantil6.

Diante deste quadro, torna-se relevante a realização de estudos que identifiquem o perfil da população atendida nos PSP, para que se qualifique a assistência prestada, minimizando os efeitos que a prematuridade causa ao desenvolvimento da criança. $O$ objetivo deste estudo foi traçar o perfil do bebê prematuro e de seus familiares assistidos em um PSP, destacando as peculiaridades da população atendida na região no período pesquisado.

\section{MÉTODO}

Trata-se de um estudo quantitativo, longitudinal que em um Ambulatório de Seguimento de Prematuros de um hospital universitário do interior do Rio Grande do Sul. Este estudo incluiu bebês e seus familiares acolhidos no PSP com idade corrigida inferior a dois anos em sua primeira consulta.

O presente estudo deriva de uma pesquisa maior que objetivou identificar se haveria impacto no desenvolvimento do bebê e no cotidiano dos pais, frente a informação sobre a prematuridade e a idade corrigida dos prematuros. Tendo em vista que havia um número reduzido de bebês com outras patologias, o que poderia interferir nos resultados do estudo amostral, definiu-se pela exclusão dos bebês com alguma patologia neurológica, erro inato de metabolismo, malformação congênita ou síndromes, pois considerou-se que poderiam interferir no crescimento e desenvolvimento do bebê, para além de questões relacionados a prematuridade.

Para traçar o perfil dos bebês e de sua família, foram coletados os dados da primeira consulta em prontuários preenchidos pela equipe médica do PSP e em questionário sociodemográfico formulado e coletado com os familiares pelas pesquisadoras. 
A coleta dos dados ocorreu de agosto de 2013 a outubro de 2015, compondo uma amostra de 92 bebês, considerando que a população total estimada foi de 110 bebês (número de vagas disponíveis para a primeira consulta no período pesquisado). As variáveis coletadas na primeira consulta em prontuário foram: sexo do neonato, peso, tipo de parto, tempo de internação da UTIN e idade gestacional. A classificação da idade gestacional para este estudo foi a definida pela World Health Organization (WHO): prematuro extremo (<28 semanas); muito prematuro (28 a $<32$ semanas) e prematuro moderado a tardio (32 a < 37 semanas) (Tradução livre dos autores). Já as variáveis obtidas do questionário sociodemográfico foram: estrutura familiar, procedência, situação de moradia, quem cuida do bebê, renda familiar e características da mãe e do pai (idade, escolaridade e situação trabalhista).

Com os dados coletados foi realizada a análise descritiva, constando de percentuais, tabelas e medidas descritivas como média, valor mínimo, valor máximo e desvio padrão. Foram utilizados os testes não paramétricos $U$ de Mann-Whitney e de independência do Qui-quadrado, sendo considerado o nível de significância de 5\%. Para a realização da análise estatística foi utilizado o aplicativo computacional Statistica 9.1.

Os dados derivam de uma pesquisa intitulada "O impacto da informação sobre a idade corrigida no desenvolvimento dos bebês prematuros e no cotidiano dos pais", aprovada pelo Comitê de Ética em Pesquisa com Seres Humanos da Universidade Federal de Santa Maria/RS, sob número CAEE 19334013.0.0000.5346, parecer 367.151, com data da Relatoria de 13/08/2013. Devido ao fato de a pesquisa maior se caracterizar como um Ensaio Clínico Randomizado, a pesquisa também foi cadastrada no Registro Brasileiro de Ensaios Clínicos sob número RBR -3dpb7f. Os pais ou responsáveis assinaram o Termo de Consentimento Livre e Esclarecido.

\section{RESULTADOS}

\section{Características dos bebês}

Da amostra composta por 92 bebês, 10,9\% nasceram de gestação múltipla. Quanto ao tipo de parto, 54,3\% nasceram de parto cesáreo, 33,7\% de parto vaginal e 12,0\% não tiveram esta informação preenchida no formulário. Quanto ao histórico gestacional, 45 (48,9\%) mães eram primíparas e destas, 50\% tiveram gestações interrompidas por aborto espontâneo. Quanto ao sexo dos bebês, 54 (58,7\%) eram do sexo feminino e 38 (41,3\%) do sexo masculino.

A idade gestacional média foi de 31,9 semanas (desvio padrão=2,9 semanas) sendo que a menor idade gestacional foi de 24,7 semanas e a maior 37,1 semanas. A média de peso ao nascer foi $1.604,5 \mathrm{~g}$ (desvio padrão=541,9g), sendo o menor peso de $705 \mathrm{~g}$ e o maior $2.955 \mathrm{~g}$. Quanto ao tempo de internação dos bebês na UTIN, a média foi de 38,4 dias (desvio padrão=23,6 dias), sendo o mínimo de cinco dias e o máximo de 127 dias. A média da idade corrigida dos bebês ao chegarem ao PSP para a primeira consulta foi de 24,6 dias, sendo o mínimo de - 47 dias 
e o máximo de 136 dias. A distribuição dos bebês pelas faixas de idade gestacional e sexo são apresentados na tabela 1. Tabela 1 - Bebês distribuídos por sexo, segundo as faixas de idade gestacional $(n=92)$

\begin{tabular}{llll}
\hline & \multicolumn{2}{c}{ Sexo (\%) } & \\
\cline { 2 - 3 } Idade gestacional (semanas) & Masculino & Feminino & Total $(\%)$ \\
\hline Extremamente prematuro $(<28)$ & $1(2,6)$ & $8(14,8)$ & $9(9,8)$ \\
Muito prematuros $(\geq 28$ e $<32)$ & $8(21,0)$ & $20(37,0)$ & $28(30,4)$ \\
Moderada a prematuro tardio $(\geq 32$ e $<37)$ & $28(73,7)$ & $26(48,2)$ & $54(58,7)$ \\
$\geq 37^{*}$ & $1(2,6)$ & $0(0,0)$ & $1(1,1)$ \\
\hline Total & $38(41,3)$ & $54(58,7)$ & $92(100,0)$ \\
\hline p-valor & \multicolumn{2}{c}{0,020} \\
\hline
\end{tabular}

*Este bebê possui idade gestacional acima do estipulado, mas peso abaixo para os critérios estabelecidos pela $\mathrm{WHO}^{7}$, devendo ser considerado prematuro. Teste U de Mann-Whitney.

Observa-se que a maioria dos bebês $(58,7 \%)$ está concentrada no nível de prematuridade considerado moderado a tardio, com idade gestacional de 32 a 36,9 semanas. Outro destaque é que, na divisão por sexo, as meninas foram prevalentes nas classificações para extremo prematuro e muito prematuro ${ }^{7}$, sendo que as idades gestacionais das meninas foram significativamente menores que a dos meninos $(p=0,020)$ (tabela 1). 0 peso também é critério para a classificação nos níveis de prematuridade, sendo os bebês distribuídos em faixas, conforme a definição da $\mathrm{WHO}^{7}$, sendo apresentados na Tabela 2.

Tabela 2 - Bebês distribuídos por sexo, segundo as faixas de peso ao nascer (OMS, 2012) (n=92)

\begin{tabular}{llll}
\hline \multirow{2}{*}{ Peso (gramas) } & \multicolumn{2}{c}{ Sexo (\%) } & \\
\cline { 2 - 3 } & Masculino & Feminino & Total $(\%)$ \\
\hline $\operatorname{RNEBP}(<1000 \mathrm{~g})$ & $2(5,3)$ & $7(13,0)$ & $9(9,8)$ \\
$\operatorname{RNMBP}(\geq 1000 \mathrm{e}<1500 \mathrm{~g})$ & $17(44,7)$ & $20(37,0)$ & $37(40,2)$ \\
$\operatorname{RNBP}(\geq 1500 \mathrm{~g} \mathrm{e}<2500 \mathrm{~g})$ & $18(47,4)$ & $22(40,7)$ & $40(43,5)$ \\
\hline$\geq 2500 \mathrm{~g}{ }^{*}$ & $1(2,6)$ & $5(9,3)$ & $6(6,5)$ \\
\hline Total & $38(41,3)$ & $54(58,7)$ & $92(100,0)$ \\
\hline p-valor & \multicolumn{3}{c}{0,631} \\
\hline
\end{tabular}

RNEBP = Recém-Nascidos de Extremo Baixo Peso; RNMBP = Recém Nascidos de Muito Baixo Peso; RNBP = Recém Nascidos de Baixo Peso. *Estes bebês possuíam peso acima do considerado como critério para prematuridade, porém sua IG é menor que 37 semanas, por isso são identificados como prematuros. Teste $U$ de Mann-Whitney 
Na tabela 2, é possível observar que o maior número de bebês se concentra na faixa de Recém-Nascido de Baixo Peso (RNBP), seguido de Recém-Nascido de Muito Baixo Peso (RNMBP), sendo que não houve diferença significativa entre os pesos de meninas e de meninos $(p=0,631)$. Na tabela 3 são apresentadas as frequências dos bebês distribuídos por faixas de peso e de idade gestacional.

Tabela 3 - Bebês distribuídos por faixas de prematuridade nas faixas de peso (OMS, 2012) (n=92)

\begin{tabular}{lcccc}
\hline & \multicolumn{4}{c}{ Idade gestacional (semanas) (\%) } \\
\hline & $\begin{array}{c}\text { Extremamente } \\
\text { prematuros } \\
\text { Peso (gramas) }\end{array}$ & $\begin{array}{c}\text { Muito } \\
\text { prematuros } \\
(<28)\end{array}$ & $\begin{array}{c}\text { Moderada a } \\
\text { prematuro tardio } \\
(\geq 28 \mathrm{e} \leq 32)\end{array}$ & $\begin{array}{c}\text { Superior } \\
\text { a 37 } 3 \mathbf{3} 37)\end{array}$ \\
\hline RNEBP $(<1000 \mathrm{~g})$ & $5(50,0)$ & $3(40,0)$ & $1(10,0)$ & $0(0,0)$ \\
RNMBP $(\geq 1000 \mathrm{~g} \mathrm{e}<1500 \mathrm{~g})$ & $3(8,1)$ & $17(45,9)$ & $17(45,9)$ & $0(0,0)$ \\
RNBP $(\geq 1500 \mathrm{~g} \mathrm{e}<2500 \mathrm{~g})$ & $1(2,5)$ & $7(17,5)$ & $31(77,5)$ & $1(2,5)$ \\
$\geq 2500 \mathrm{~g}$ & $0(0,0)$ & $1(16,7)$ & $5(83,3)$ & $0(0,0)$ \\
\hline Total & $9(9,8)$ & $28(30,4)$ & $54(58,7)$ & $1(1,1)$ \\
\hline
\end{tabular}

RNEBP = Recém-Nascidos de Extremo Baixo Peso; RNMBP = Recém Nascidos de Muito Baixo Peso; RNBP = Recém Nascidos de Baixo Peso

Os resultados da tabela 3 são esperados, pois quanto maior a idade gestacional, maior será o crescimento do bebê, haja vista que 31 dos bebês considerados prematuros moderados a tardio, são também RNBP. Somente um bebê desta faixa é Recém-Nascido de Extremo Baixo Peso (RNEBP).

\section{Características dos familiares}

Para essas famílias, os cinco pares de bebês gemelares tiveram seus pais duplicados, visto que cada bebê, participante deste estudo, foi considerado de forma individual. Em relação ao local de residência das famílias, $57,1 \%$ residem na cidade sede do hospital pesquisado e 42,9\% são das cidades da região de sua abrangência. Quanto à situação de moradia, 56,5\% vivem em casa própria, 31,5\% vivem em casa alugada, 7,6\% vivem em casa emprestada e 3,3\% se enquadraram na opção "outros".

Considerando a situação conjugal dos pais $68,2 \%$ estavam em união estável, 18,1\% eram casados, 9,1\% solteiros, 2,3\% divorciados e 2,3\% consideraram-se na opção "Outros". Sobre os cuidados com o bebê, $15,9 \%$ informaram que este papel é assumido somente pela mãe, $42,0 \%$ informaram que os pais ajudam no cuidado, $25,0 \%$ informaram que os avós ajudam no cuidado, 8,0\% são outros não familiares que ajudam no cuidado, 3,4\% informaram que todos ajudam no cuidado e 5,7\% não informaram. 
$\mathrm{Na}$ tabela 4, são apresentadas medidas descritivas da idade, escolaridade e situação trabalhista das mães e pais dos bebês.

Tabela 4 - Mães e pais conforme a idade, situação trabalhista e escolaridade ( $n=92)$

\begin{tabular}{lllll}
\hline & & Mãe $(\mathrm{n}=91)$ & Pai $(\mathrm{n}=87)$ & p-valor \\
\hline Idade (anos) & Média (desvio padrão) & $26,2(6,8)$ & $30,0(7,6)$ & $0,001^{*}$ \\
\hline & Mínimo-máximo & $15,0-50,0$ & $17,0-53,0$ & \\
\hline Trabalha & Sim & $34(37,0 \%)$ & $77(83,7 \%)$ & $<0,001^{\text {** }}$ \\
\hline Escolaridade & Superior completo & $5(5,5 \%)$ & $2(2,3 \%)$ & 0,867 \\
& Superior incompleto & $5(5,5 \%)$ & $5(5,8 \%)$ & \\
& Médio completo & $26(28,6 \%)$ & $32(37,2 \%)$ & \\
& Médio incompleto & $15(16,5 \%)$ & $10(11,6 \%)$ & \\
& Fundamental completo & $21(23,1 \%)$ & $13(15,1 \%)$ & \\
& Fundamental incompleto & $19(20,9 \%)$ & $24(27,9 \%)$ & \\
\hline
\end{tabular}

Um pai não informou a escolaridade; *Teste U de Mann-Whitney; ** Teste de independência do Qui-quadrado;

Na tabela 4, observa-se que as idades das mães são significativamente inferiores em relação às dos pais. Quanto ao trabalho, há um número maior de pais que possuem emprego formal se comparados às mães. Em relação à escolaridade, não houve diferença significativa entre mães e pais, destacando que é pequeno o número, tanto de pais quanto de mães, com ensino superior completo ou incompleto. A renda média mensal familiar foi de três salários mínimos (desvio padrão=1,0 salário mínimo), com mínimo de um e máximo de seis salários, sendo que no período da pesquisa, o valor correspondente ao salário mínimo era de $\mathrm{R} \$ 678,00$.

\section{DISCUSSÃO}

O Programa de Seguimento de Prematuros, constituído por equipes multiprofissionais, foi criado como uma estratégia para diminuir os impactos da prematuridade no desenvolvimento dos bebês. A premissa de que tanto o bebê quanto seus familiares devem ser atendidos neste programa, amplia a assistência ultrapassando a visão unívoca da realização de uma avaliação, orientação e encaminhamento voltado, somente, para a condição clínica do bebê (paciente). A existência de uma equipe multiprofissional (terapeutas ocupacionais, fisioterapeutas, fonoaudiólogos, psicólogos, serviço social e especialidades médicas), deve servir para que o plano de intervenção seja, ao mesmo tempo singular e também de âmbito global quanto às necessidades do bebê e de sua família ${ }^{8}$.

Este plano de intervenção pode ser compreendido tanto como o acompanhamento do desenvolvimento do bebêe seus riscos, quanto como o acolhimento e orientação às dúvidas e angústias dos pais frente às respostas do bebê, muitas 
vezes diminuídas pela condição imposta pela prematuridade e pelos efeitos produzidos pela internação prolongada em UTIN . Somado a isso, deve-se considerar também a condição socioeconômica da família e as características culturais da região, mesmo que a assistência local tenha como base de orientação um programa proposto por instâncias superiores, a nível governamental, por exemplo9.

\section{Perfil do bebê atendido no PSP}

Neste estudo, as meninas constituíram a maioria $(58,7 \%)$ dos nascimentos prematuros. Nas pesquisas de Marcuartú e Malveira ${ }^{10}$ e Buffone, Eickman, Lima², as meninas também foram a maioria, com 73\% e 57,4\%, respectivamente. Porém, esses resultados diferem de outro estudo encontrado na literatura como o de Silva et al. ${ }^{8}$, que obtiveram percentuais maiores de meninos $(60,3 \%)$.

Com relação à idade gestacional, a prematuridade do público alvo dessa pesquisa teve maior concentração na classificação de prematuridade de moderado a tardio (58,7\%). Já, o número de prematuros extremos foi o menor (9,8\%). Da mesma forma, os autores Melo e Carvalho ${ }^{11}$, Buffone, Eickman e Lima², em seus estudos, categorizaram a maioria dos bebês estudados como prematuros moderados a tardios, com $86 \%$ e $65 \%$, respectivamente. Em relação aos recém nascidos prematuros extremos, apenas $0,2 \%$ dos bebês do estudo de Melo e Carvalho ${ }^{11}$ foram classificados nessa categoria, diferente do estudo de Marcuartú e Malveira ${ }^{10}$, que obtiveram uma grande quantidade de bebês classificados na categoria de prematuridade extrema, alcançando um percentual de $73 \%$.

Já, quanto à classificação por peso, considerando o resultado esperado de que bebês com maior idade gestacional tenham maior peso, o que chama a atenção é o cruzamento entre os bebês considerados na faixa de prematuridade de moderada a tardio em relação aos seus pesos. De um total de $58,7 \%$ de bebês de prematuridade moderada a tardia, 45,9\% são considerados RNMBP, resultado igual aos bebês na faixa de muito prematuros (tabela 3). Estes dados confirmam a importância de o PSP garantir assistência ampliada para além da idade gestacional, considerando também o peso do bebế. Para este estudo, os bebês classificados como RNEBP são em número pequeno (9,8\%) (tabela 2), mas nem por isso são menos importantes. Quando distribuídos por idade gestacional, os RNEBP concentram-se no grupo de extremos prematuros com cinco bebês, muito prematuro com três e moderado a tardio com um bebê (tabela 3).

Mesmo não representando a maior porcentagem, esses resultados indicam que existem bebês com maior risco à mortalidade e ao atraso no desenvolvimento neuropsicomotor. É importante levar em conta a especificidade da clientela atendida, pois dela se definirá também o nível de investimento em recursos materiais e humanos.

Outra questão que pode ser agregada às características do bebê é o tempo de internação na UTIN. Os cuidados na UTIN têm grande relevância para a sobrevida dos bebês prematuros e para o seu desenvolvimento ${ }^{12}$, diminuindo os 
índices de complicações ${ }^{13}$. Os bebês participantes desta pesquisa permaneceram em média 38,4 dias internados na UTIN. Resultado semelhante ao da pesquisa de Abrahão et al. ${ }^{14}$ que apresentou a média de permanência dos bebês na UTIN de 31 dias e no estudo de Marcuartú e Malveira ${ }^{10}$ com média de 43,8 dias.

O estudo de Santos ${ }^{15}$ sobre a influência da idade gestacional no desenvolvimento motor, ressalta que os baixos escores no desenvolvimento de pré-termo estão diretamente associados à admissão em UTIN. Marcuartú e Malveira ${ }^{10}$ apontam que esse tempo maior de hospitalização pode gerar consequências no crescimento e desenvolvimento dos bebês, devido a fatores presentes cotidianamente nas UTIN que causam estresse para o bebê, como: luminosidade, sons intensos, excesso de manipulação e repouso inadequado ${ }^{10}$.

Outro fator importante que impacta no tempo de gestação é o tipo de parto. Neste grupo, dos 81 bebês com informações sobre o parto, 54,3\% nasceram de parto cesáreo. É preciso considerar que o hospital, campo desta pesquisa, é referência na região para gestantes de alto risco, aumentando a chance de que o parto seja cesáreo e prematuro. Estudos apontam que a prevalência de prematuridade é maior entre nascidos de parto cesáreo. Entre eles destacam-se as pesquisas realizadas em hospitais públicos do Brasil ${ }^{10,16,17,18} \mathrm{e}$ as pesquisas realizadas com os dados do Datasus, do Sistema de Informações sobre Nascidos Vivos (Sinasc) ${ }^{19,20}$. No entanto, Carvalho et al. ${ }^{21}$, Carvalho et al. ${ }^{22}$, Santos et al. ${ }^{15}$ e Silva et al. ${ }^{8}$ em pesquisas realizadas também em hospitais públicos, encontraram em seus estudos maior frequência de partos vaginais associados a nascimentos prematuros.

\section{Perfil da família atendida no PSP}

Em relação às características sociodemográficas da família, a maioria dos pais se encontrava em união estável ou eram casados e as famílias viviam, na maioria, em casa própria ou alugada. O estado civil dos pais foi problematizado em outra pesquisa como a de Assunção et al. ${ }^{23}$, cujos resultados não apresentaram diferenças significativas entre 0 número de mães solteiras e casadas, que em sua maioria, encontraram uma porcentagem alta de mães que possuem companheiro $8,16,21,22$, sendo também o caso deste estudo.

Para esta pesquisa, o cuidado do bebê era realizado pela mãe e pai em $42,0 \%$ dos casos, representando um número maior em relação as mulheres que referiram cuidar sozinhas do bebê (15,9\%). Nas consultas médicas dos bebês, era comum estarem presentes a mãe e o pai. Esse dado é importante para se pensar no impacto das informações repassadas na consulta, pois, há necessidade de se estabelecer um diálogo horizontalizado entre a equipe do PSP e a mãe e o pai, a fim de se considerar as singularidades das famílias, reconhecendo suas necessidades, valorizando seus saberes e os corresponsabilizando pelos projetos estabelecidos para o desenvolvimento do filho ${ }^{24}$.

Quanto à renda salarial mensal das famílias, o valor médio de três salários mínimos é maior do que o encontrado na pesquisa de Carvalho et al..$^{22}$ que apontam, em seu estudo com bebês pré-termo, uma porcentagem média de 64,2\%, 
que recebiam de um a dois salários mínimos. Assim como no estudo de Carvalho et al. ${ }^{21}$, que apresentou $76,2 \%$ das famílias com uma renda menor ou igual a um salário mínimo.

Em relação à escolaridade, os estudos encontrados apresentam dados somente em relação às mães. Nesta pesquisa, $28,6 \%$ das mães possuem ensino médio completo, seguidas de $23,1 \%$ que possuem ensino fundamental completo e 20,9\% com ensino fundamental incompleto. Menetrier e Almeida ${ }^{16}$ também encontraram maior número de mães com ensino médio completo. No entanto, outros estudos destacam que estes dados são preocupantes ${ }^{8,17,20}$, já que na classificação do Ministério da Educação, o ensino médio deveria acontecer entre os 15 e 17 anos para todos/as os/as jovens brasileiros. Neste sentido a escolaridade materna de bebês prematuros, segundo Oliveira et al. ${ }^{20}$ é considerada "inadequada" (p.384) aos padrões pré-estabelecidos pelo sistema educacional.

Estes dados podem ajudar a problematizar a construção de um diálogo entre a equipe de saúde (normalmente com ensino superior e pós-graduação) e o paciente (com escolaridade inferior ao estabelecido como ideal), colocando em questão, novamente a necessidade de se compreender a cultura regional e utilizá-la como recurso para estabelecer diálogos cujos pais sintam-se implicados nos cuidados do filho prematuro, para além da orientação médica.

Os resultados encontrados por esta pesquisa em relação à idade materna apontam uma média de 26,2 anos, aproximando-se de resultados encontrados por outros autores $8,15,18,19,25,26$. No entanto, $24,1 \%$ delas tiveram seus bebês em idades consideradas de risco: 8,0\% tiveram seus bebês antes dos 18 anos e 16,1\% tiveram seus bebês com 34 anos ou mais. Atualmente, alguns estudos afirmam que a idade materna é um fator de risco para o nascimento prematuro $8,14,16,18,19,20,21,23,26$.

Por fim, é importante destacar que quase metade, $42,9 \%$ dos bebês atendidos são oriundos de outros municípios na região de abrangência do PSP, exigindo dos pais e da equipe ações em rede de assistência que extrapolam a instituição hospitalar, esbarrando nas dificuldades enfrentadas pelos pequenos municípios com relação à assistência de média e alta complexidade.

\section{CONSIDERAÇÕES FINAIS}

A pesquisa confirma a necessidade de uma análise regionalizada, com vistas a buscar a implementação de políticas públicas que acolham as necessidades locais, através da formação continuada de profissionais e no acolhimento, educação e informação dos pais quanto a seus direitos e responsabilidade, assegurados pelo Sistema Único de Saúde (SUS).

Esta é uma informação importante para se levar em conta nos futuros investimentos tanto no que tange a equipamentos/instrumentos de avaliação, formação dos profissionais que compõem a equipe, quanto para ações 
que subsidiem o planejamento e execução de políticas públicas de saúde. Contribui para a melhoria da qualidade da assistência prestada, pautada pela singularidade, mas também pela necessidade de compreender-se o bebê e seus familiares a partir de diferentes aspectos.

\section{CONTRIBUIÇÃO DOS AUTORES}

${ }^{1,2}$ Coleta e análise dos dados, concepção do texto manuscrito, organização dos dados e das fontes e/ou análises e redação do texto, aprovação da versão final a ser publicada, responsável por todos os aspectos do trabalho.

${ }^{3,4}$ Orientadora do estudo: concepção da pesquisa, da análise dos dados, do texto manuscrito, organização de fontes e/ou análises e revisão, aprovação da versão final a ser publicada, responsável por todos os aspectos do trabalho.

${ }^{5}$ Coorientadora do estudo: Concepção e delineamento do estudo, análise estatística e revisão dos dados e do texto, aprovação da versão final a ser publicada, responsável por todos os aspectos do trabalho.

\section{REFERÊNCIAS}

1. Lawlor GCO, Righi NC, Kurtz FM, Porto BSS, Trevisan CM. Caracterização de variáveis clínicas e do desenvolvimento motor de recém nascidos prematuros. Rev. APS. 2018 abr - jun; 21(2): 177 - 181. Disponível em: file://C:/Users/Vitoria/Downloads/Prematuridade/Caracterização\%20de\%20variáveis\%20clínicas.pdf. Acesso em: 18 de fev. 2019

2. Buffone AFRRC, Eickman BSH, Lima BMC. Processamento sensorial e desenvolvimento cognitivo de lactentes nascidos pré-termo e a termo. Cad. Ter. Ocup. UFSCar. 2016. 24(4): 695-703. ISSN 0104-4931. http://dx.doi. org/10.4322/0104-4931.ctoA00731. Disponível em: <http://www.cadernosdeterapiaocupacional.ufscar.br/index.php/cadernos/article/view/1384/769>. Acesso em: 05 de jan. 2018.

3. Maggi EF, Magalhães LC, Campos AF, Bouzada MCF. Crianças pré-termo apresentam desempenho motor, cognitivo e funcional desfavorável em relação a neonatos a termo em idade pré-escolar. J Pediatr. 2014 jul - ag. 90 (4): 377-383. Disponível em: <http://dx.doi.org/10.1016/j.jped.2013.10.005 >. Acesso em: 22 de nov. 2017. 
4. Zerbeto AB, Cortelo FM, Filho EBC. Associação entre idade gestacional e peso ao nascimento no desenvolvimento linguístico de crianças brasileiras: revisão sistemática. 2015 jul - ag. J Pediatr. 91 (4): 326-332. Disponível em:<http://dx.doi.org/10.1016/j.jped.2014.11.003>. Acesso em: 20 de nov. 2017.

5. Secretaria Estadual de Saúde - RS.Plano Estadual de Saúde: 2016/2019. Porto Alegre: Secretaria da saúde. 2016. Disponível em: <http://www.saude.rs.gov.br/upload/arquivos/201701/05153251-pes-2016-2019-sesrs.pdf>. Acesso em: 04 de jan. 2018.

6. Brasil. Ministério da Saúde. Portaria n 1459, de 24 de junho de 2011. Institui, no âmbito do Sistema Único de Saúde - SUS - a Rede Cegonha. Diário Oficial [da] República Federativa do Brasil. Brasília, DF, Seção 1, 24 de jun. 2011. Disponível em: <http://bvsms.saude.gov.br/bvs/saudelegis/gm/2011/prt1459_24_06_2011.html>. Acesso em: 04 mai. 2018.

7. World Health Organization. Born Too Soon: The Global Action Report on Preterm Birth.Geneva. 2012. Disponível em: <www.who.int/pmnch/media/news/2012/201204_borntoosoon-report.pdf>. Acesso em: 05 de jan. 2018.

8. Silva ES, Filho FRAM, Soares RFS, Carvalho, FM.O perfil socioeconômico e epidemiológico de mães e recém-nascidos pré-termo. 2017 abr - jun. R. Interd. 10 (2): 47-57. Disponível em: <https://revistainterdisciplinar.uninovafapi.edu.br/index.php/revinter/article/view/1085>. Acesso em: 05 de jan. 2018.

9. Peruzzolo DL, Estivalet KM, Mildner AR, Silveira MC. Participação da Terapia Ocupacional na equipe do Programa de Seguimento de Prematuros Egressos de UTINs. 2014. Cad. Ter. Ocup. UFSCar. Disponível em: http://www. cadernosdeterapiaocupacional.ufscar.br/index.php/cadernos/article/view/976/499. Acesso em: 05 de jan.2018.

10. Marcuartú AC, Malveira SS. Perfil de Recém-Nascidos Prematuros de Muito Baixo Peso Internados em Unidade de Cuidados Intensivos Neonatais. 2017. R. Bras. Ci. Saúde. 21 (1): 5-10. Disponível em: <http://periodicos. ufpb.br/ojs2/index.php/rbcs/article/view/28551/16429>. Acesso em: 05 de jan. 2018.

11. Melo WA, Carvalho MDB. Análise multivariada dos fatores de risco para a prematuridade no Sul do Brasil. Rev Eletrônica Gestão \& Saúde. 2014. 5(02): 1-10. Disponível em: <http://periodicos.unb.br/index.php/rgs/article/ view/22773/16321>. Acesso em: 05 de jan. 2018. 
12. Beltrame V, Moraes A, Souza AP. Perfil sensorial e sua relação com risco psíquico, prematuridade e desenvolvimento motor e de linguagem por bebês de 12 meses. Rev. Ter. Ocup. [Internet]. 2018. 29 (1): 8-18. [citado 23fev.2019];29(1):8-8. Available from: http://www.revistas.usp.br/rto/article/view/138550.

13. Oliveira CS, Casagrande GA, Grecco LC, Golin MO. Perfil de recém-nascidos pré-termo internados na unidade de terapia intensiva de hospital de alta complexidade. ABCS. 2015. 40(3): 229-233. Disponível em: < https:// www.portalnepas.org.br/abcshs/article/view/700> Acesso em: 21 de fev. 2019.

14. Abrahão CAF, Silva KR, Castro GG. Perfil do recém-nascido no serviço terapêutico de acompanhamento precoce. Journal of Amazon Health Science 2015. 1(2): 64-84. Disponível em: <http://revistas.ufac.br/revista/index.php/ ahs/article/view/243/pdf_12>. Acesso em: 04 jan. 2018

15. Santos GA, Vieira MEB, Linhares MBA, Formiga CKMR. Influência da idade gestacional no desenvolvimento motor de bebês pré-termo de 0 a 6 meses de idade corrigida. In: III Congresso de ensino pesquisa e extensão da UEG. Inovação: Inclusão social e direitos. Outubro de 2016. Disponível em: < file:///C:/Users/ACER/Downloads/6806-Texto\%20do\%20artigo-20271-1-10-20161003.pdf > Acesso em: 20 de fev. 2019.

16. Menetrier JV, Almeida, G. Perfil epidemiológico de gestantes de alto risco com parto prematuro em um hospital de referência. Rev. Saúde e Pesquisa. set./dez. 2016. 9(3): 433-441. DOI: http://dx.doi.org/10.177651/19831870.2016. Disponível em: <http://periodicos.unicesumar.edu.br/index.php/saudpesq/article/view/5534/2926>. Acesso em: 05 de jan. 2018.

17. Gonzaga ICA, Santos SLD, Silva ARV. Atenção pré-natal e fatores de risco associados à prematuridade e baixo peso ao nascer em capital do nordeste brasileiro. Ciência \& Saúde Coletiva. 2016. 21(6):1965-1974. DOI: 10.1590/1413-81232015216.06162015. Disponível em: <http://www.scielo.br/pdf/csc/v21n6/1413-8123-csc-21-06-1965. pdf>. Acesso em: 05 de jan. 2018.

18. Tabile PM, Teixeira RM, Toso G, Matras RC, Fuhrmann IM, Pires MC, et al. Características dos partos pré-termo em hospital de ensino do interior do Sul do Brasil: análise de 6 anos. Rev AMRIGS. 2016. 60(3): 168-172. Disponível em: <http://www.amrigs.org.br/revista/60-03/02_1523_Revista\%20AMRIGS.PDF>. Acesso em: 05 de jan. 2018. 
19. Balbi B ,Carvalhaes MA de BL, Parada CMGL. Tendência temporal do nascimento pré-termo e de seus determinantes em uma década. Ciência \& Saúde Coletiva. 2016. 21(1): 233-241. DOI: 10.1590/141381232015211.20512015. Disponível em: <http://www.scielo.br/pdf/csc/v21n1/1413-8123-csc-21-01-0233.pdf>. Acesso em: 05 de jan. 2018.

20. Oliveira LL, Gonçalves AC, Costa JSD, Bonilha ALL. Fatores maternos e neonatais relacionados à prematuridade. Rev. Esc. Enferm. 2016. 50(3): 382-389. DOI: http://dx.doi.org/10.1590/S0080-623420160000400002. Disponível em: <http://www.scielo.br/pdf/reeusp/v50n3/pt_0080-6234-reeusp-50-03-0382.pdf>. Acesso em: 05 de jan.2018.

21. Carvalho SS, Coelho JMF, Bacelar DA, Mariola E. Fatores maternos para o nascimento de recém-nascidos com baixo peso e prematuros: estudo caso-controle. Ciência \&Saúde 2016. 9(2): 76-82. Disponível em: <http:// revistaseletronicas.pucrs.br/ojs/index.php/faenfi/article/view/21947/14826>. Acesso em: 05 de jan. 2018.

22. Carvalho JBL, Teixeira GA, Morais PC, Sena AV, Alves TR de M. Condições socioeconômicas da gestação de bebês prematuros. Rev. enferm. UFPE. 2018. Fev. 12(2): 386 - 390. Disponível em: <https://periodicos.ufpe.br/ revistas/revistaenfermagem/article/viewFile/15294/27840> Acesso em: 20 de fev. 2019

23. Assunção PL, Novais HMD, Alencar GP, Melo ASO, Almeida MF. Fatores associados ao nascimento pré-termo em Campina Grande, Paraíba, Brasil: um estudo caso-controle. Cad. Saúde Pública. 2012. 28(6): 1078-1090. Disponível em: <http://www.scielo.br/pdf/csp/v28n6/07.pdf> Acesso em: 04 jan. 2018.

24. Kalichman AO, Ayres JRCM. Integralidade e tecnologias de atenção à saúde: uma narrativa sobre contribuições conceituais à construção do princípio da integralidade no SUS. Cad. Saúde Pública. 2016. ago. 32(8): 01-13. Disponível em: <http://www.scielo.br/scielo.php?pid=S0102311X2016000803001\&script=sci_abstract\&tlng=pt>. Acesso em: 05 de jan. 2018.

25. Machado AKF, Marmitt LP, Cesar JA. Prematuridade tardia no extremo sul do Brasil: um estudo de base populacional. Rev. Bras. Saúde Matern. Infant. 2016 abr / jun.16 (2): 121-128. Disponível em: < http://www.scielo.br/ scielo.php?pid=S1519-38292016000200113\&script=sci_arttext\&tlng=pt > Acesso em: 20 fev. 2019.

26. Guimarães EAA, Vieira CS, Nunes FDD, Januário GC, Oliveira VC, Tibúrcio JD. Prevalência e fatores associados à prematuridade em Divinópolis, Minas Gerais, 2008-2011: análise do Sistema de Informações sobre Nascidos Vivos.Epidemiol. Serv. Saúde. 2017 jan-mar. 26(1): 91-98. doi: 10.5123/S1679-49742017000100010. Disponível em: <http://www.scielo.br/pdf/ress/v26n1/2237-9622-ress-26-01-00091.pdf>. Acesso em: 05 de jan.2018. 